\title{
Cloudy with a chance of Pain
}

\author{
Adapted from "Weather Patterns \\ Associated with Pain in Chronic-Pain \\ Sufferers," by David M. Schultz \\ (University of Manchester), Anna \\ L. Beukenhorst, Belay Birlie Yimer, \\ Louise Cook, Huai Leng Pisaniello, \\ Thomas House, Carolyn Gamble, \\ Jamie C. Sergeant, John McBeth, \\ and William G. Dixon. Published on- \\ line in BAMS, May 2020. For the full, \\ citable article, see DOI:10.1175 \\ /BAMS-D-19-0265.1.
}

A common belief among three-quarters of those who suffer from chronic pain is that their pain fluctuates with the weather. However, this belief lacks scientific support. In a recent review of 41 studies examining the relationship between weather and chronic musculoskeletal pain, 28 (68\%) found some relationship, although there was disagreement about what that relationship was.

Various reasons have been postulated for the disagreement: small sample sizes as measured by the number of participants, the duration of the study, or both; weather observations that were unrepresentative of conditions experienced by participants; and the lack of input or analysis by meteorologists.

To overcome these limitations of previous studies, we designed a 15-month-long U.K.-based citizen-science smartphone project called Cloudy with a Chance of Pain (www.cloudywithachanceofpain .com). Participants with chronic pain used a specially designed app to enter a 10-question daily report on their pain and other well-being characteristics on a 5-point scale. For example, participants rated their pain from 1 ("no pain") to 5 ("very severe pain").

The absolute number in a participant's report may not be meaningful, by itself: participants reporting the same severity on our 5-point scale may experience different levels of pain. Studies show, however, that a $20 \%$ increase in pain severity is clinically significant. As such, we defined a pain event for an individual participant when they report a 1-category or greater increase $(+1)$ in their pain level from the previous day.

Using the global positioning system (GPS) sensor in the phone, we linked the participants' locations during the study to the closest weather stations in the Met Office observing network. Thus, we developed a daily profile of the average weather conditions each 

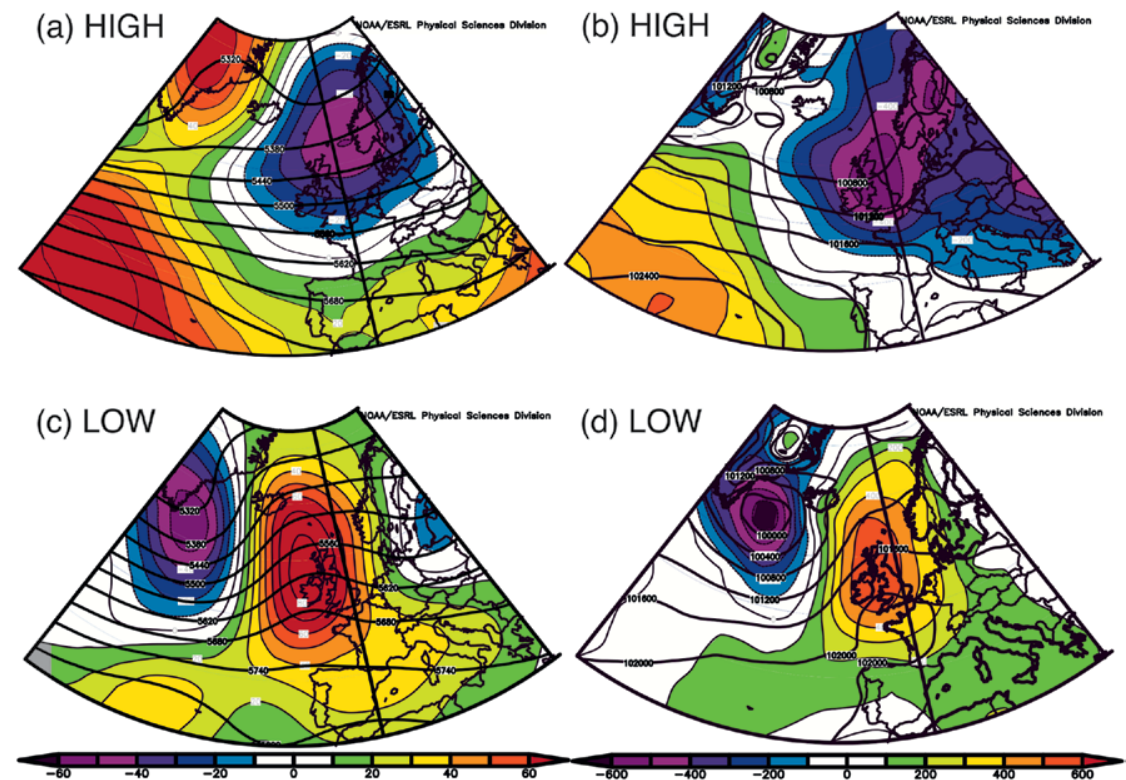

500-hPa mean geopotential height and anomalies (m) mean sea-level pressure and anomalies $(\mathrm{Pa})$ participant experienced within the United Kingdom.

In a previous study, we performed an independent analysis of the dataset using a case-crossover design, an epidemiological method that compares, for each individual, days with a pain event to control days without a pain event, thus controlling for all time-invariant participant characteristics. The analysis, based on a much smaller dataset, demonstrated statistically significant-albeit modest-relationships between high pain events and high relative humidity, low sea level pressure, and high wind speed. Temperature and precipitation did not have statistically significant relationships with pain. These results held up even when accounting for mood and physical activity.

Here, we see if these results are reproducible from a meteorological perspective, using synoptic climatology and compositing to visualize the synoptic-scale weather patterns associated with pain.

\section{Synoptic composites of HIGH and $*>$}

LOW days. Anomalies computed relative to the weighted average of the daily 1981-2010 means.
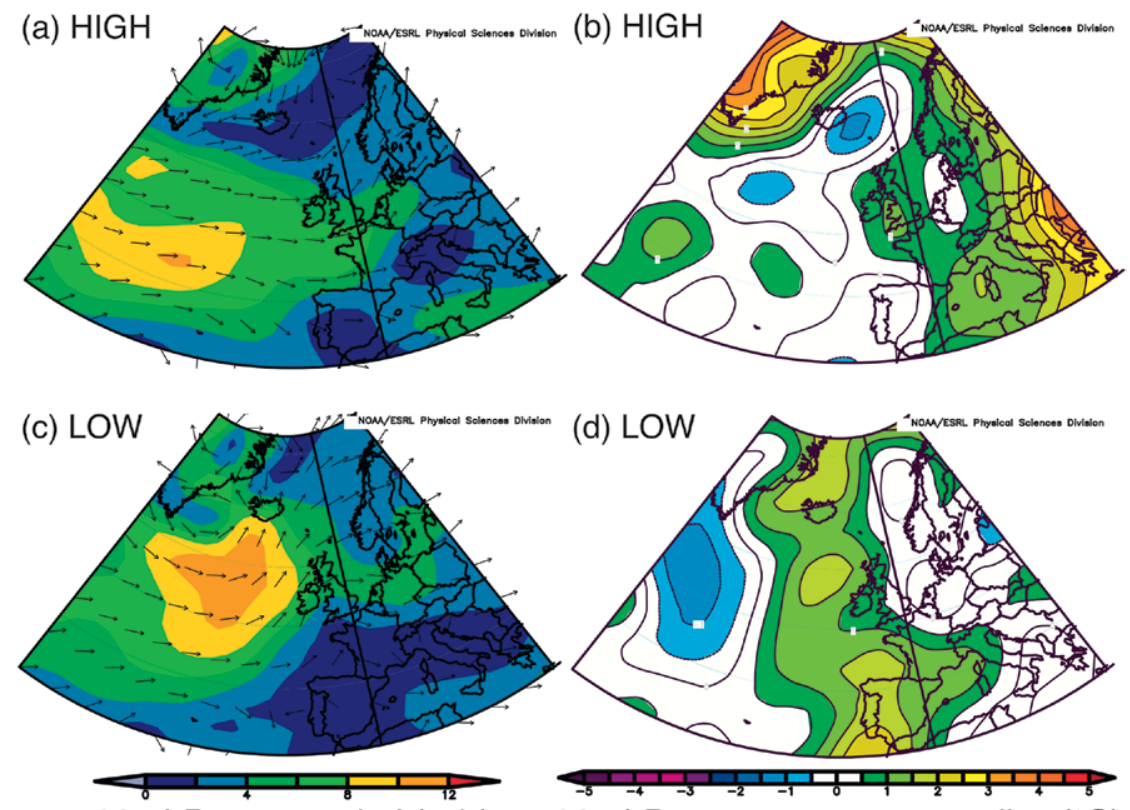

925-hPa mean wind $(\mathrm{m} / \mathrm{s})$
$4 *$ Synoptic composites of HIGH and LOW days. Anomalies computed relative to the weighted average of the daily 1981-2010 means.

\section{Data and methods}

The study ran from 20 January 2016 to 19 April 2017. Bolstered by national media attention, 13,207 users throughout the United Kingdom downloaded the study app at some point during a 12-month recruitment period. A total of 10,584 participants entered their demographic information and at least one pain report, making them eligible for the study. Sixty-five percent of participants remained in the study beyond their first week and $44 \%$ remained beyond their first month. Even after 200 days, $15 \%$ of participants remained consistent contributors. The exceptionally high rate of retention likely reflects the easy-to-use app design, as well as the dedication of participants to contribute to answering a question of great personal interest to them.

In all, there were 445 days available for study. The top 10\% (45 days) with the highest percentage of participants having a +1 or greater pain event were termed HIGH. The bottom $10 \%$ with the lowest percentage of 

were termed LOW. The most painful days (HIGH) had $23 \%$ of participants across the United Kingdom reporting an increase in pain, and the least painful days (LOW) had only 10\% of participants reporting an increase in pain. Hourly weather observations were averaged to produce daily weather conditions for each individual who submitted daily pain reports as well as U.K.-average weather for the day.

\section{Relating pain to weather}

Most HIGH days occurred from January to June 2016, whereas most LOW days occurred from June 2016 to January 2017. Variations by month in the fraction of participants experiencing a pain event suggest that seasonal changes in weather might affect levels of pain, although we could not discount that the population of active participants changed over the course of the study.

The 1200 UTC weather maps for each HIGH and LOW day were averaged together to produce synoptic composites. Detailed composite analysis of 500-hPa geopotential height, sea level pressure, precipitation, $925-\mathrm{hPa}$ moisture, wind, and temperature reveal remarkably different weather patterns during HIGH versus LOW days.

On HIGH days, the jet stream was aimed right at the United Kingdom. This is associated with below-normal (or low) pressure, and thus more wind, moisture, and precipitation. In contrast, on LOW days, the jet stream tended to blow north of the United Kingdom, bringing above-normal (or high) pressure and associated below-normal humidity and precipitation rates, and weaker winds. In short, the results provide the strongest scientific support for people who have been saying that the weather affects their pain but who have not been taken seriously by other people, their own doctors included.

\section{Discussion}

Although the results are intriguing, they apply only to the United Kingdom. Similar studies should be replicated elsewhere to see if these findings can be generalized. Another caveat is that many participants had multiple health conditions causing pain, and even those with the same underlying condition may have felt pain differently. We didn't group the participants into different disease categories in our participants having a +1 or greater pain event

analysis-a topic of future research. Also, untangling the effect of those who stayed inside-due to severe pain or other reasonsrequires further analysis.

Although specific weather patterns may not be the primary cause of people's pain, our results demonstrate that weather does modulate pain in at least some individuals. Who is most susceptible remains to be determined.

People have been talking about the effect of weather on their pain for millennia, so why is this particular research project important? One reason is that our study had both the longest duration and the most participants of all previous studies, allowing greater confidence in the fidelity of our results. Another reason is that this research compares for the first time the weather patterns on days with a large number of people reporting pain and days with a low number reporting pain. Finally, our research begins to shed light on the environmental conditions that modulate pain, insight that might be explored further for improving the treatment, management, and forecasting of pain. $\therefore$

BAMS: What would you like readers to learn from this article?

\section{David M. Schultz (University of Manchester): \\ Three-quarters of people who suffer from pain report that they believe that weather influences their pain-yet their concerns are often dismissed by others, including their doctors. Although weather may not be the primary influence on people's pain, we show that it can modulate pain events in some people. Our study gives patients more support for their beliefs, showing that their beliefs have validity.}

BAMS: How did you get involved in this project?

DMS: Project lead Prof. William Dixon had heard anecdotal reports of the influence of weather on pain from his patients in clinic. He was working on using mobile technology to better collect information on patient's symptoms in between visits to the clinic when he realized that the GPS sensor in mobile phones could be linked to the closest weather station to record weather data closest to the patients. A collaboration with uMotif, a company that creates mobile health 


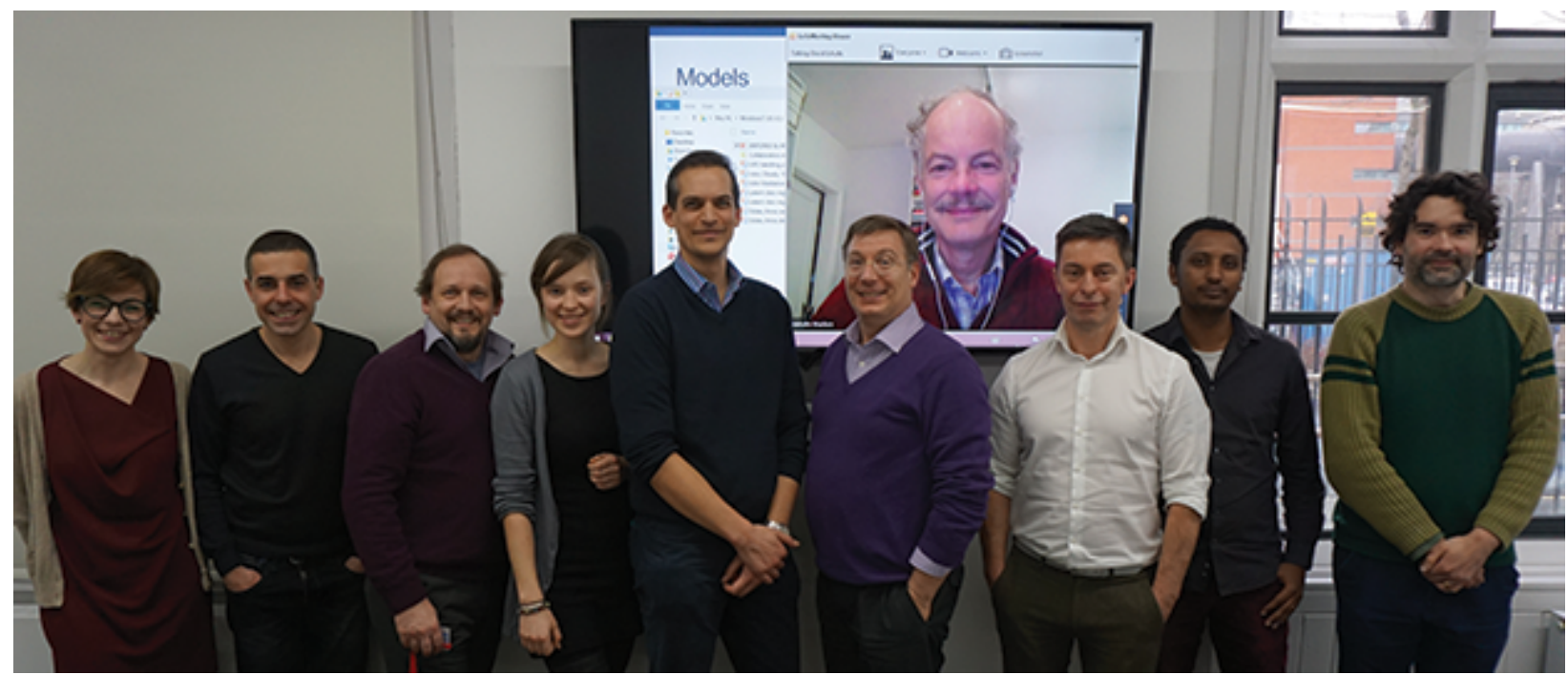

$\Delta$

* Some of the main research team on the Cloudy With a Chance of Pain project: Ana M. Vicedo-Cabrera, Antonio Gasparrini, Mark Lunt, Anna L. Beukenhorst, William G. Dixon, David M. Schultz, Malcolm Maclure (on screen), John McBeth, Belay B. Yimer, and Ricardo Silva.

apps, led to the app to collect patient data. Thus, William could envision a research project that would test out ideas about engagement with health apps and answer a longstanding question about the effect of weather on pain. A chance meeting between William and my Head of Department brought me on board.

BAMS: What attracted you to the idea of relating weather to chronic pain?

DMS: I had a latent interest in weather and pain because both of my parents have arthritis. I jumped at the opportunity to get involved in the project. It's been one of the most satisfying research projects of my career.

BAMS: What surprised you the most about the work you document in this article?

DMS: The enthusiasm of the citizen scientists who entered their data on a daily basis. When we designed the project, we had hoped we might get 1,000 people to sign up. However, William appeared on the $B B C^{\prime}$ 's Trust Me I'm a Doctor and on
BBC Breakfast. Because of the media interest, and the enthusiastic and financial support of Versus Arthritis (formerly Arthritis Research UK), we had over 13,000 people download the app. Shortly after William's appearance on BBC Breakfast, people were signing up to the study at a rate of one per second, testing the capacity/resilience of the server at uMotif. And these people were enthusiastic about participating! Most people download apps, use them a few times, and then stop. Other studies of engagement with mobile apps confirm such low retention rates of participants with apps. However, even after six months into our study, $15 \%$ of participants were still entering their data nearly every day. The success of this study is due to their dedication. We are so grateful for their participation.

BAMS: What about surprises in the results?

DMS: We had expected a weak relationship and maybe some insight into the weather-pain relationship. The strength of the relationship and its robustness were surprising.
BAMS: What was the biggest challenge in the project?

DMS: The principal challenge was rapidly scaling up our project from a small funded pilot study of 20 people to a national-scale project. Although we were excited that the opportunity to be on $B B C$ television would help promote our project nationally and boost recruitment, we were challenged by finding a source of funding to build the app and pay for data collection and hosting on such a short time scale. That meant from the time of recording the show to when it aired, we had six months to get funding for a national-scale project. Fortunately, Versus Arthritis was open to considering a proposal with such a short time frame, and they provided enough funding from them to carry out the data collection. The issue of data analysis was another story. In that case, other synergies (e.g., incoming Ph.D. student and postdoctoral researcher, collaborations with other researchers at other institutions) happened later that allowed us to bring a diverse group of people onto the team who could help analyze the data. 